\title{
Control of a Hyper-Redundant Robot for Quality Inspection in Additive Manufacturing for Construction
}

\author{
Othman Lakhal $^{1}$, Achille Melingui ${ }^{2}$, Gerald Dherbomez $^{1}$ and Rochdi Merzouki ${ }^{1}$
}

\begin{abstract}
Additive manufacturing is an automated process for producing layer-by-layer material deposition. Recently this technology has been introduced in the industrial construction in order to print houses or smaller piece structures for on-site assembly, with complex geometry. In Additive manufacturing processes, the material deposition step is generally followed by a printing quality inspection step. However, the geometry of printed structures with minimal surfaces is sometimes complex, where rigid structure robots cannot reach certain zones to scan their surfaces. In this paper, a continuum-hyper-redundant manipulator equipped with a camera is attached to the endeffector of a mobile-manipulator robot for the quality inspection process. Indeed, Continuum manipulators can bend along structures with complex geometry; and this inherent flexibility makes them suitable for navigation and operation in congested environments. The number of controlled actuators being greater than the dimension of task space, this work is summarized in a trajectory tracking of hyper-redundant robots. This issue lies in the resolution of strongly nonlinear equations with a realtime computation. Thus, a hybrid methodology which combines the advantages of quantitative and qualitative approaches is used for modeling and resolution of the hyper-redundant robot kinematics. A kinematic controller was designed and a set of experiments was carried out to evaluate the level of efficiency of the proposed approach.
\end{abstract}

\section{INTRODUCTION}

Additive manufacturing in the building sector is an emerging construction technique, which is inspired by methods similar to $3 \mathrm{D}$ printing, i.e. the production of sequentially stratified objects [1]. In addition, additive manufacturing for buildings is seen as an innovative, economical, affordable and environmentally friendly technique. It can also increase the efficiency, the safety in the work and significantly reduce the construction time [2]. So far, three additive manufacturing techniques have been developed; Contour Crafting [3], DShape [4], and Concrete Printing [5]. A comparison study was proposed in [6]. These techniques minimize the duration of the construction process in comparison with conventional methods.

Additive manufacturing robots open new perspectives for manufacturing and construction. Nowadays, many approaches have been applied such as manufacturing on site or in the laboratory, using different technologies. Some use Cartesian robots to print prefabricated parts in the laboratory

\footnotetext{
*This work was not supported by Matrice project

Othman Lakhal, Gerald Dherbomez and Rochdi Merzouki are with Polytech Lille, CRIStAL, CNRS-UMR 9189, Avenue Paul Langevin, 59655 Villeneuve d'Ascq, France rochdi.merzouki@polytech-lille.fr.

${ }^{2}$ Achille Melingui is with the Department of Electrical and Telecommunications Engineering, University of Yaounde 1, Yaounde 8390, Cameroon achillemelingui@gmail.com.
}

and transport them to the site afterward. For example, WinSun Decoration Design Engineering has succeeded in building houses from construction components printed in prefabricated elements and assembled on site [7]. Others use a gantry system like WinSun [8] or TotalKustom [9]. Nevertheless, these systems have limited workspace and are often complicated or slow to implement. Other researchers have designed a machine to automate and facilitate the setup and printing process. The IAAC University of Barcelona [10] has designed a family of mobile robots for small-scale construction and easy to install. Each robot must perform a diverse task, which slows down the printing process and limits the architectural form to print. Recently, the emergence of mobile robots (rolling or flying) is increasingly present in the field of additive manufacturing in the building like [11]. The use of mobile robots for 3D printing combines the advantages of gantry and robot handling systems by printing on the outside and inside. But also, to employ multiple mobile robots printing concurrently a large, single-piece, structure [11].

Moreover, it is essential to inspect the quality of the printed or repaired structure during or after the manufacturing phases. This monitoring phase can be automated to detect the appearance of crack, have a return to the quality of the surface, and check the adhesion between the printed layers. Several systems have been developed to automate the inspection process in some specific environments, such as subway tunnel [12], flexible pavement surfaces [13], [14], bridge decks [15] and building [16],[17]. In [18], a hexapod robot, named Hex-piderix, with a stereo camera has been used to inspect the cracking on surfaces like walls or roofs of a building. Access to these places is sometimes very difficult for people. Some use Unmanned Aerial Vehicles (UAVs) systems in the visual inspection of buildings. The crack detection is essential for health monitoring of built infrastructure. An integrated system that enables revisiting crack locations during building inspections by means of a quadrature UAV is presented in [19]. In [20], a UAV used for the data collection in order to create a 3D model of the structure by using laser scanners. Thanks to $3 \mathrm{D}$ printing and 3D scanning, it is possible to check the impression quality and repair very complex details. However, the geometry of the printed structures, with funicular surfaces can sometimes be complex as traditional rigid structure robots cannot reach certain critical areas of the printed structure.

Recently, Continuum-style robots, which can bend at any point along their structure have emerged. This inherent flexibility makes them suitable for various applications, in- 
cluding navigation and operation in complex and congested environments [21], [22]. In this work, this flexibility has been exploited to navigate through complex printed structures during the quality inspection process.

As the mobile manipulator carries a soft manipulator equipped with a camera for scanning the printed structure, the control objective comes down to trajectory tracking of the hyper-redundant robot composed of a mobile rigid manipulator and an extended continuum arm. In recent decades, a lot of research has been done on redundant mobile manipulators, which has spawned three important methods, namely the extended Jacobian [23], the projected gradient, and the operational space extension method [24], these methods also optimize secondary objectives for avoiding singularities, joint angle limits or increasing manipulability [25]. In this work, the kinematic equations of the hybrid structure are first derived, and an Optimal solution is obtained by using a quadratic program, thereafter. The challenging control task is the control of the of the soft manipulator. Indeed, in our previous work, [26], [27], [28], we have referred to the need to introduce adaptive algorithms to cope with undesirable effects of Compact Bionic Handling Assistant (CBHA). We have further noted that the use of non-adaptive control laws is not sufficient as the robot model changes over time. Therefore, we were accorded to an update of the robot model and real-time adjustment of the controller's parameters. In this work, the high-accuracy of the KUKA manipulator is exploited to improve the performance of the CBHA. Once the desired shape is obtained using an open-loop kinetic controller, the remaining errors, if they exist, are reduced by using the inverse kinematics of the manipulator. This greatly reduces the computation time in real time.

The remaining structure of the document is as follows: Section 2 presents the description of the experimental platform, i.e. a mobile manipulator equipped with a flexible manipulator. Section 3 develops the kinematic equations of the experimental platform and their resolution via the quadratic program while section 4 focuses on controlling the hybrid structure robot. The real-time implementation and results obtained are presented in section 5 and some concluding observations are provided in section 6 .

\section{DESCRIPTION OF THE ROBOTIC PLATFORM}

The experimental platform called MATRICE's robot is a prototype for additive manufacturing in construction. It is composed of a mobile Omni-drive platform, an industrial rigid manipulator (KUKA), and a soft arm, as shown in Fig 1 . The idea is to design a prototype with an industrial scale that can move and navigate autonomously in the construction site or inside confined spaces. The holonomic mobile platform embeds sensors for localization, namely: wheel encoders and an inertial measurement unit (IMU). It facilitates the maneuvering and installation of the robot on site and can cover a large workspace for printing or scanning. A six degrees of freedom (DoF) manipulator robot which carries a soft manipulator for the quality inspection is mounted on the mobile platform. During the scanning phase, a 3D camera is attached to a pneumatic manipulator (Fig. 2), namely CBHA designed by FESTO-DIDACTIC. The choice comes from the fact that it can maneuver in congested and narrow places thanks to its flexibility. We note that easily accessible areas may require complex movements of rigid platforms with the risk of reaching joint limits. The use of flexible manipulators not only reduces the scanning time but also the energy consumed.

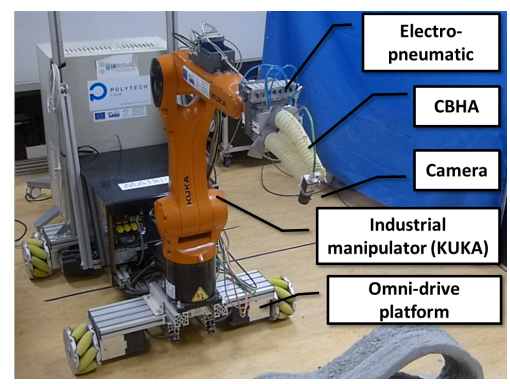

Fig. 1: Description of MATRICE robot

The considered bionic-continuum arm in this work is called CBHA, which looks like an elephant's trunk (Fig. 2) or a backbone. Each flexible section consists of three tubes, controlled by electro-pneumatic actuators. The position and orientation of each section are controlled by varying the pressure inside tubes. The system is controlled by six actuators (three per flexible sections). Six tubes can be loaded and unloaded with compressed air so that the structure can be moved in any desired direction. Six wire-potentiometers sensors are placed along each tube allowing to measure elongations of each tube. The voltages provided by wirepotentiometer sensors are proportional to the extension of each tube $l_{p, s}$, where $p=1, \ldots, 3$ and $s=1,2$ correspond to the wire-potentiometer and section number, respectively.

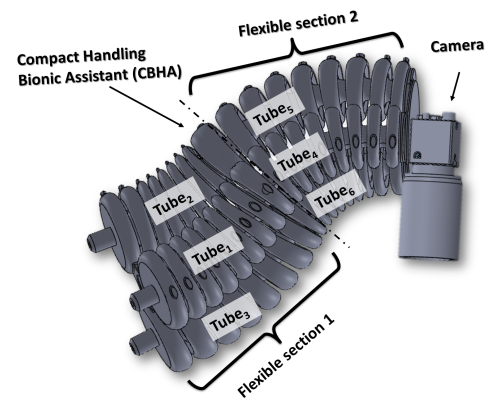

Fig. 2: Description of the CBHA platform

The mobile manipulator and the electro-pneumatic of the CBHA are controlled by a real-time controller, namely the CompactRio of National Instruments. Indeed, the input/output modules have been configured to communicate with the different actuators and sensors of the robot. However, the manipulator is outfitted with I/O cards to allow direct access to the controller in real-time. An interface namely KUKA Ethernet RSI (Remote Sensor Interface) communicates between the controller's hardware of the 
rigid manipulator and the CompactRio, through a real-time network link. Data are transmitted through the UDP/IP protocol. This external connection is based on XML data that must be exchanged in less than $12 \mathrm{~ms}$, and represents the communication cycle time.

\section{HYPER-REDUNDANT ROBOTIC PLATFORM KINEMATICS}

This section develops the kinematic equations of the hybrid and hyper-redundant robot structure.

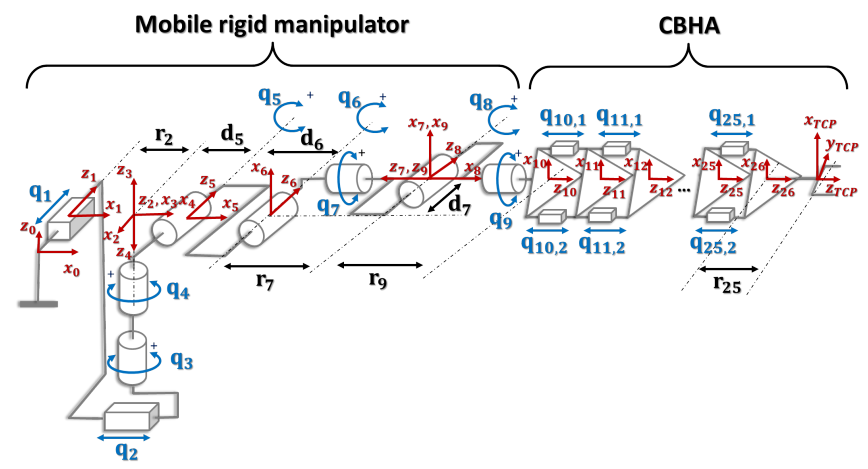

Fig. 3: Placement of frames in robot home position

The kinematic equations of the soft-continuum manipulator and the rigid mobile manipulator are successively presented.

\section{A. Kinematics of the soft-continuum manipulator CBHA}

In our previous work, the kinematics of the CBHA has been developed [27]. The designed controller was able to guide the CBHA's end effector towards the desired target within 6-mm positioning accuracy. The modeling approach was inspired by the modeling concept of parallel manipulators. The CBHA was modeled as a series of vertebrae, where each vertebra was connected to the next one through a flexible link. The latter named an inter-vertebra was modeled by three universal prismatic-spherical joints and one universalprismatic joint, Fig. 4 Under this consideration, the CBHA is can be viewed as a concatenation of 16 discrete parallel robots i.e, a rigid parallel robot of 48-DoF.

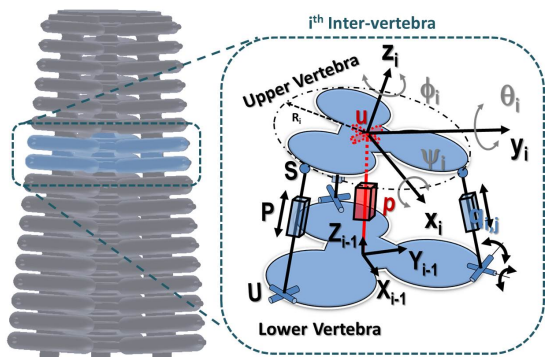

Fig. 4: An inter-vertebra modelled as parallel robot with 3UPS-1UP

The elegance of the proposed approach was the possibility to derive the entire continuum robot kinematic only from the inverse kinematics equation (IKEs) of one inter-vertebra.

$$
\begin{aligned}
q_{i, 1}^{2}= & \left.Z_{i}^{2}+2 R_{i} Z_{i} S \theta_{i}-2 R_{i} R_{i-1} C \theta_{i}+R_{i-1}^{2}+R_{i}^{2}\right) \\
q_{i, 2}^{2}= & Z_{i}^{2}+Z_{i} R_{i}\left(\sqrt{3} C \theta_{i} S \psi_{i}-S \theta_{i}\right)+R_{i}^{2}+R_{i-1}^{2} \\
& -R_{i} R_{i-1}\left(\frac{\sqrt{3}}{2} S \theta_{i} S \psi_{i}+\frac{3}{2} C \psi_{i}+\frac{1}{2} C \theta_{i}\right) \\
q_{i, 3}^{2}= & Z_{i}^{2}-Z_{i} R_{i}\left(\sqrt{3} C \theta_{i} S \psi_{i}+S \theta_{i}\right)+R_{i}^{2}+R_{i-1}^{2} \\
& +R_{i} R_{i-1}\left(\frac{\sqrt{3}}{2} S \theta_{i} S \psi_{i}-\frac{3}{2} C \psi_{i}-\frac{1}{2} C \theta_{i}\right)
\end{aligned}
$$

Note that $S$ and $C$ refer to sine and cosine functions, respectively. These equations (1) are obtained by calculating the joint variables $q_{i, j}$ corresponding to the position $Z_{i}$ and the orientation $\theta_{i}, \psi_{i}$ of the upper vertebra's frame relative to the lower vertebra frame, where $j=1, . ., 3$ is the number of active joints and $i=10, . ., 25$ is the number of joint link, as shown in Fig. 3. In addition, the entire shape of the CBHA is conical, i.e. the radii of the upper $R_{i}$ and lower $R_{i-1}$ vertebrae are different.

The process of inverse kinematic modeling of the CBHA is summarized in Fig. 5. Indeed, for a given desired endeffector pose; the middle of both sections is approximated from a neural network (NN). The position $Z_{i}$ and the orientation $\theta_{i}, \psi_{i}$ of the set of upper vertebrae are approximated from three neural networks. Finally, the elongations of each inter-vertebra are obtained through the IKES. For more details, we refer the interested reader to [27], [29].

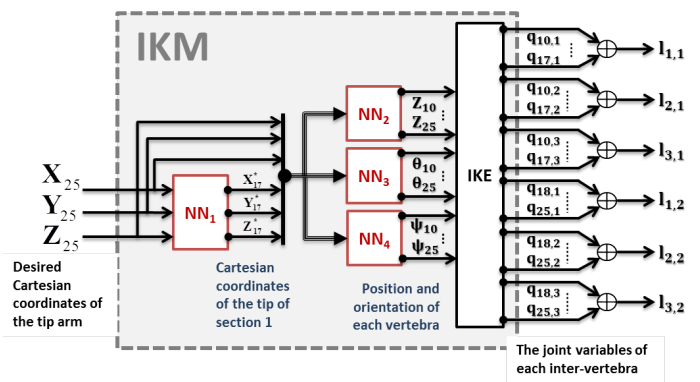

Fig. 5: Algorithm for inverse kinematic modelling

\section{B. Kinematics of the mobile-manipulator}

In this work, we are confronted with the inverse kinematic problem of hyper-redundant mobile manipulators, where an infinite number of configuration vectors is possible for a given Cartesian position. In the $3 \mathrm{D}$ printing process, the resolution of redundancies is essential because the environment is constantly changing, and printed structures, for example, become obstacles. But the abundance of degrees of freedom leads to increasing complexity, which makes it difficult to ensure precise control of the system. In addition, various phenomena such as slippages accentuate this error multiplication effect. Thus, this problem can be addressed from a kinematic point of view by exploiting the performance of each subsystem and thus choosing the solution that optimizes the system's behavior. The idea is to minimize the number of movements of the mobile platform during trajectory tracking in order to reduce location errors.

The Mobile manipulator robot, depicted in Fig. 3 is characterized by a six degree of freedom arm and three degrees 
of freedom mobile-platform. Thus, in a first step, analytical equations of the forward kinematic model is obtained using the Khalil-Kleinfinger method [30], namely the modified Denavit-Hartenberg (D-H) [31] Convention.

TABLE I: D-H parameters for hyper-redundant robot

\begin{tabular}{|c|c|c|c|c|c|c|}
\hline Link & $\sigma_{i}$ & $\mu_{i}$ & $\alpha_{i}$ & $r_{i}$ & $\theta_{i}$ & $d_{i}$ \\
\hline 1 & 1 & 1 & $-\pi / 2$ & $q_{1}$ & $-\pi / 2$ & 0 \\
\hline 2 & 1 & 1 & $-\pi / 2$ & $q_{2}$ & $\pi / 2$ & 0 \\
\hline 3 & 0 & 0 & $\pi / 2$ & 0 & $-q_{3}+\pi / 2$ & 0 \\
\hline 4 & 0 & 0 & $\pi$ & 0 & $q_{4}$ & 0 \\
\hline 5 & 0 & 0 & $\pi / 2$ & 0 & $q_{5}$ & $d_{5}$ \\
\hline 6 & 0 & 0 & 0 & 0 & $q_{6}-\pi / 2$ & $d_{6}$ \\
\hline 7 & 0 & 0 & $-\pi / 2$ & $r_{7}$ & $-q_{7}$ & $d_{7}$ \\
\hline 8 & 0 & 0 & $-\pi / 2$ & 0 & $-q_{8}$ & 0 \\
\hline 9 & 0 & 0 & $-\pi / 2$ & 0 & $q_{9}$ & 0 \\
\hline
\end{tabular}

The matrix ${ }^{i-1} T_{i}$, where $i$ is the joint number, is expressed according to the parameters mentioned in the above table $\mathrm{I}$. Thus, by substituting the different transformation matrices, the transformation of the frame $\mathfrak{R}_{9}$ with respect to the frame $\mathfrak{R}_{0}$ is obtained :

$$
\underbrace{{ }^{0} T_{3}\left(q_{1}, q_{2}, q_{3}\right)}_{\text {Mobile platform }} \cdot \underbrace{{ }^{3} T_{9}\left(q_{4}, q_{5}, q_{6}, q_{7}, q_{8}, q_{9}\right)}_{\text {Rigid manipulator }}=u
$$

with $u$ the desired position :

$$
u=\left(\begin{array}{cccc}
n_{x} & o_{x} & a_{x} & P_{x} \\
n_{y} & o_{y} & a_{y} & P_{y} \\
n_{z} & o_{z} & a_{z} & P_{z} \\
0 & 0 & 0 & 1
\end{array}\right)
$$

Thus, from equation (2), we can derive the following equations:

$$
\left\{\begin{aligned}
P_{x}= & C_{34} S_{56} d_{7}+C_{34} C_{56} r_{7}+C_{34}\left(C_{5} d_{6}+d_{5}\right)+q_{2} \\
P_{y}= & -S_{34} S_{56} d_{7}-S_{34} C_{56} r_{7}-S_{34}\left(C_{5} d_{6}+d_{5}\right)+q_{1} \\
P_{z}= & C_{56} d_{7}-S_{56} r_{7}-S_{5} d_{6} \\
n_{x}= & \left(\left(C_{34} S_{56} C_{7}+S_{34} S_{7}\right) C_{8}+C_{34} C_{56} S_{8}\right) C_{9} \\
& -\left(C_{34} S_{56} S_{7}-S_{34} C_{7}\right) S_{9} \\
n_{y}= & \left(\left(-S_{34} S_{56} C_{7}+C_{34} S_{7}\right) C_{8}-S_{34} C_{56} S_{8}\right) C_{9} \\
& -\left(-S_{34} S_{56} S_{7}-C_{34} C_{7}\right) S_{9} \\
n_{z}= & \left(C_{56} C_{7} C_{8}-S_{56} S_{8}\right) C_{9}-C_{56} S_{7} S_{9} \\
o_{x}= & -\left(\left(C_{34} S_{56} C_{7}+S_{34} S_{7}\right) C_{8}+C_{34} C_{56} S_{8}\right) S_{9} \\
& -\left(C_{34} S_{56} S_{7}-S_{34} C_{7}\right) C_{9} \\
o_{y}= & -\left(\left(-S_{34} S_{56} C_{7}+C_{34} S_{7}\right) C_{8}-S_{34} C_{56} S_{8}\right) S_{9} \\
& -\left(-S_{34} S_{56} S_{7}-C_{34} C_{7}\right) C_{9} \\
o_{z}= & -\left(C_{56} C_{7} C_{8}-S_{56} S_{8}\right) S_{9}-C_{56} S_{7} C_{9} \\
a_{x}= & \left(C_{34} S_{56} C_{7}+S_{34} S_{7}\right) S_{8}-C_{34} C_{56} C_{8} \\
a_{y}= & \left(-S_{34} S_{56} C_{7}+C_{34} S_{7}\right) S_{8}+S_{34} C_{56} C_{8} \\
a_{z}= & C_{56} C_{7} S_{8}+S_{56} C_{8}
\end{aligned}\right.
$$

Thus, to solve equations (4), we proceed in two steps; first, we calculate the joint variables for the omnidrive mobile platform in presence of redundant equations, in order to obtain the optimal configuration. Then, the obtained variables $q_{1}, q_{2}$ and $q_{3}$, are used to calculate the joint variables of the rigid manipulator $\left(q_{4}, q_{5}, q_{6}, q_{7}, q_{8}\right.$ and $\left.q_{9}\right)$ by using
Paul's method [32]. However, for quality inspection, some manipulator configurations are unnecessary or to be avoided. For example, singular configurations, including those that cause a collision with a part of the robot (platform, electrical cabinet, etc.), should be avoided. Thus, these constraints relative of the mechanical limits of the manipulator.

These constraints lead to a sub-workspace of the rigid manipulator. The latter reduces greatly the number of inverse kinematic solutions. By substituting the constraints into the first two equations of the system 4 the inequality can be reformulated as follows 5

$$
\begin{aligned}
& X_{\max } \geq P_{y} S_{3}-q_{1} S_{3}+P_{x} C_{3}-q_{2} C_{3} \geq X_{\min } \\
& Y_{\max } \geq-P_{x} S_{3}+P_{y} C_{3}-q_{1} C_{3}+q_{2} S_{3} \geq Y_{\min }
\end{aligned}
$$

where $X_{\max }, X_{\min }, Y_{\max }$ et $Y_{\min }$ represent the boundaries of the sub-workspace. The equation 6 represents the objective function adopted in the optimization procedure. Optimal displacements of the mobile platform are done using a quadratic program (QP) [33]. This approach makes it possible to minimize a quadratic objective function under a finite number of inequality constraints. The equation 6 represents the objective function adopted in the optimization procedure.

$$
\min _{Q} \frac{1}{2} \mathbf{Q}^{T} H \mathbf{Q}+g^{T} \mathbf{Q} \text { such that }\left\{\begin{array}{l}
A \cdot \mathbf{Q} \leq \mathbf{b} \\
A e q \cdot \mathbf{Q}=\mathbf{b e q} \\
\mathbf{l b} \leq \mathbf{Q} \leq \mathbf{u b}
\end{array}\right.
$$

where $\mathbf{Q}=\left[q_{1}, q_{2}\right]$ represents the linear displacements of the mobile platform. The orientation $q_{3}$ is obtained by exploiting the normal to the curve at each point of the path.

\section{COnTROL MATRICE Robot}

The control of the MATRICE robot takes place in two stages, namely an off-line and on-line step. The off-line step calculates the trajectories of the configuration vectors from the desired trajectory of the TCP. For real-time constraints and in order to improve the tracking accuracy, the desired TCP trajectory is divided into two trajectories. The first trajectory tracked by the mobile manipulator makes it possible to approach the effector of the structure to be scanned. The second trajectory tracked by the CBHA manipulator allows scanning of the structure. The goal is to exploit the KUKA manipulator's precise tracking capability to compensate for CBHA faults.

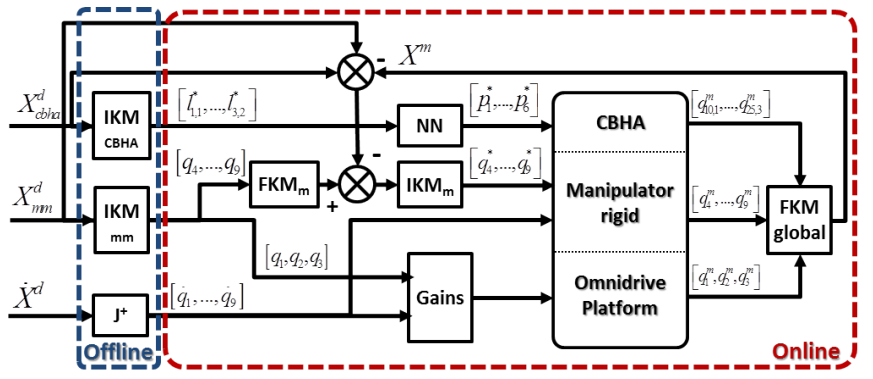

Fig. 6: Kinematic control scheme 


\section{A. Generation of joint-space trajectories: off-line step}

In this work, the desired trajectory is generated from slicing data used during de printing phase. Indeed, the slicing process is an operation of producing a $G$-code file from a 3D model file, in preparation for 3D printing. The $G$-code is converted to the robot coordinates, thereafter. Therefore, from the Cartesian coordinates of one slice, it is possible to generate the desired TCP trajectory. This trajectory is moved so as to have a large field of view of the camera allowing the scanning of the inner surface of the object, as shown in Fig 7

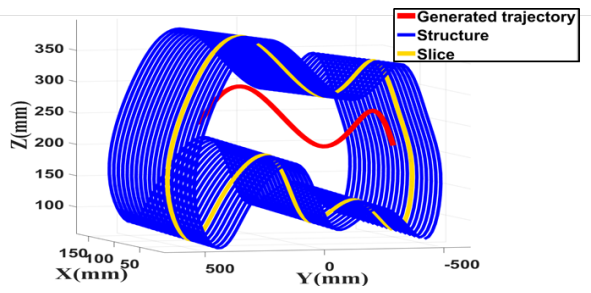

Fig. 7: Trajectory generation

The intermediate trajectory corresponding to the positioning of the frame $\{9\}$ relative to the frame $\{0\}$ is set not only to place the camera as close as possible to the scanning structure but also so that the desired TCP trajectory is inside the CBHA's workspace. Therefore, the placement of the frame for each point of the intermediate trajectory is based on an approach based on the placement of Bishop frames [34], in order to avoid the appearance of twists. The Bishop frame vectors are generated on a spatial curve using the algorithm proposed in [35]. The placement of the frame allows knowing the position and orientation of the CBHA's base.

During the inspection process, it necessary to avoid all possible collisions with obstacles. Since the optimization approach only selects one inverse kinematic solution among the redundancies manifolds, the obstacle avoidance is performed offline through a series of simulation tests. Indeed, during simulation tests, the trajectory generated by the endeffector of the CBHA and the corresponding CBHA's shapes are displayed, and Configurations leading to a collision with the structure are eliminated for the benefit of others.

\section{B. Control of the hyper-redundant robot: the on-line step}

The control architecture is depicted in Fig. 6. It consists of an offline and online part. The offline part provides the desired configuration vector trajectories, namely the joint variable trajectories of the mobile manipulator by $\mathrm{IKM}_{m m}$ and pseudo-inverse $j^{+}$, and the elongation vector trajectories of the CBHA by $\mathrm{IKM}_{c b h a}$. The desired elongations of the CBHA is convert to pressures. The control signals feed the omnidrive platform, the manipulator rigid and the CBHA simultaneously. The rigid manipulator and the electro-pneumatic for the CBHA, have their specific PID controller. A PID controller has been established to track the desired trajectory of the omnidrive platform. The output provided by the $\mathrm{FKM}_{\text {global }}$ bloc is compared with the desired
TCP trajectory. The $\mathrm{IKM}_{m}$ of the rigid manipulator is then used to reduce the Cartesian errors.

\section{IMPLEMENTATION AND EXPERIMENT RESULTS}

This section presents the experiments conducted and the results obtained. It starts with the localization of the mobile platform and follows with the results obtained. The section ends with a discussion.

\section{A. Experiment results}

The obtained and desired trajectories from the robot sensors are represented in Fig 8a We observe that the endeffector of the CBHA tracks the desired trajectory. Euclidean errors of the TCP of the CBHA with respect to the desired trajectory are given in Fig $8 \mathrm{~b}, 8 \mathrm{c}$, and $8 \mathrm{~d}$. The range of the Euclidean error is approximately $\left[\begin{array}{ll}-10 & 10\end{array}\right] \mathrm{mm}$. The implementation video can be viewed on the following link: https://www.youtube.com/watch?v=BdPqfLp0png

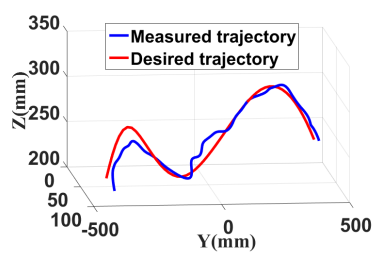

(a) Trajectories

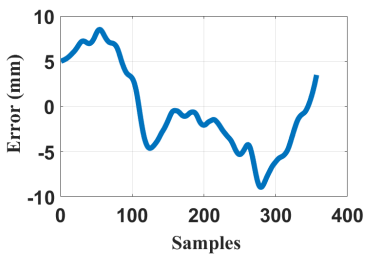

(c) Euclidean error in $\mathrm{Y}$ axis;

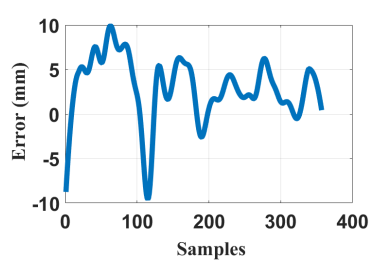

(b) Euclidean error in $\mathrm{X}$ axis;

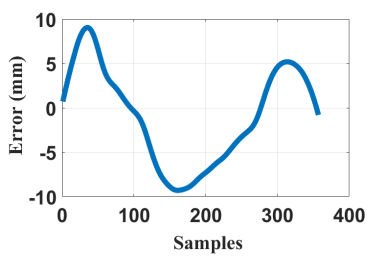

(d) Euclidean error in $\mathrm{Z}$ axis;
Fig. 8: Trajectory tracking by the hyper-redundant robot

\section{B. Discussions}

In view of the obtained results, the proposed kinematic controller achieves suited performances in terms of tracking trajectories in the area of the construction. The 3D visualization of the CBHA posture makes it possible to assess the quality of the inverse solution obtained, in particular as regards the avoidance of obstacles. The TCP positioning error is less than $10 \mathrm{~mm}$, which is reasonable for scanning operations. The purpose of this work was to enable rapid scanning and segmentation of the complex geometry of printed objects with additive manufacturing technique. The pictures capture will allow an offline processing of the structure quality. Indeed, online processing will slow down the scanning process. Euclidean errors can be explained by the drift of Swedish wheels during the displacement of the mobile platform. Remaining small Cartesian errors are expected to result from the memory effects and nonstationary behaviors of the soft-continuum arm (CBHA). 


\section{CONCLUSIONS}

In this paper, a control strategy is developed for trajectory tracking of a hyper-redundant robot for quality inspection in the additive manufacturing process for construction. This robotic platform consists of a holonomic mobile platform, an industrial rigid-manipulator, and a soft-continuum arm. The latter was considered for handling a camera for external scanning of complex printing objects. The proposed kinematic controller was derived from the kinematic equations of the robotic platform. The resolution of the latter was performed by means of a quadratic program. The real-time experiments were conducted and the results obtained were satisfactory according to the specifications and requirements of scanning operations in Construction area. In future work, the orientation of the camera may be considered for control purpose, and the CBHA's shape may be integrated into control architecture for real-time obstacle avoidance. The attached camera may be utilized to improve tracking performance.

\section{ACKNOWLEDGEMENT}

This research work has been conducted within the MATRICE project (www.matrice-3dprinting.com) which was cofinanced by the European Regional Development Fund and the "les Hauts-de-France" region in France.

\section{REFERENCES}

[1] J. B. Gardiner and S. R. Janssen, "Freefab - development of a construction-scale robotic formwork 3d printer," in Robotic Fabrication in Architecture, Art and Design 2014, pp. 131-146, Springer, 2014.

[2] R. Mathur, "3d printing in architecture," International Journal of Innovative Science, Engineering \& Technology, vol. 3, 2016.

[3] B. Khoshnevis, A. Carlson, and M. Thangavelu, "Isru-based robotic construction technologies for lunar and martian infrastructures," 2017.

[4] V. Colla and E. Dini, "Large scale 3d printing: From deep sea to the moon," Low-Cost 3D Printing, for Science, Education \& Sustainable Development; Canessa, E., Fonda, C., Zennaro, M., Eds, pp. 127-132, 2013.

[5] S. Lim, R. A. Buswell, T. T. Le, R. Wackrow, S. A. Austin, A. G. Gibb, and T. Thorpe, "Development of a viable concrete printing process," 2011.

[6] I. Perkins and M. Skitmore, "Three-dimensional printing in the construction industry: A review," International Journal of Construction Management, vol. 15, no. 1, pp. 1-9, 2015.

[7] I. Hager, A. Golonka, and R. Putanowicz, " $3 \mathrm{~d}$ printing of buildings and building components as the future of sustainable construction?," Procedia Engineering, vol. 151, pp. 292-299, 2016.

[8] Winsun, "Website of the company winsun : 3d printing architecture's future." http://www.winsun3d.com, 2014. visited on 30 august 2018.

[9] D. D. Camacho, P. Clayton, W. O'Brien, R. Ferron, M. Juenger, S. Salamone, and C. Seepersad, "Applications of additive manufacturing in the construction industry-a prospective review," in ISARC. Proceedings of the International Symposium on Automation and Robotics in Construction, vol. 34, Vilnius Gediminas Technical University, Department of Construction Economics \& Property, 2017.

[10] MiniBuilder, "Website of the iaac university : Minibuilder." robots.iaac.net/, 2013. visited on 30 august 2018.

[11] X. Zhang, M. Li, J. H. Lim, Y. Weng, Y. W. D. Tay, H. Pham, and Q.-C. Pham, "Large-scale 3d printing by a team of mobile robots," Automation in Construction, vol. 95, pp. 98-106, 2018.

[12] W. Zhang, Z. Zhang, D. Qi, and Y. Liu, "Automatic crack detection and classification method for subway tunnel safety monitoring," Sensors, vol. 14, no. 10, pp. 19307-19328, 2014.

[13] H. Oliveira and P. L. Correia, "Automatic road crack detection and characterization," IEEE Transactions on Intelligent Transportation Systems, vol. 14, no. 1, pp. 155-168, 2013.
[14] Y. Shi, L. Cui, Z. Qi, F. Meng, and Z. Chen, "Automatic road crack detection using random structured forests," IEEE Transactions on Intelligent Transportation Systems, vol. 17, no. 12, pp. 3434-3445, 2016.

[15] P. Prasanna, K. J. Dana, N. Gucunski, B. B. Basily, H. M. La, R. S. Lim, and H. Parvardeh, "Automated crack detection on concrete bridges.," IEEE Trans. Automation Science and Engineering, vol. 13, no. 2, pp. 591-599, 2016.

[16] R.-J. Yan, E. Kayacan, I.-M. Chen, and L. K. Tiong, "A novel building post-construction quality assessment robot: Design and prototyping," in Intelligent Robots and Systems (IROS), 2017 IEEE/RSJ International Conference on, pp. 6020-6023, IEEE, 2017.

[17] I.-M. Chen, E. Asadi, J. Nie, R.-J. Yan, W. C. Law, E. Kayacan, S. H. Yeo, K. H. Low, G. Seet, and R. Tiong, "Innovations in infrastructure service robots," in ROMANSY 21-Robot Design, Dynamics and Control, pp. 3-16, Springer, 2016.

[18] J. Z. De Paz, E. C. Castañeda, X. S. Castro, and S. R. Jimenez, "Crack detection by a climbing robot using image analysis," in Electronics, Communications and Computing (CONIELECOMP), 2013 International Conference on, pp. 87-91, IEEE, 2013.

[19] F. Kucuksubasi and A. Sorguc, "Transfer learning-based crack detection by autonomous uavs," arXiv preprint arXiv:1807.11785, 2018.

[20] M. D. Phung, V. T. Hoang, T. H. Dinh, and Q. Ha, "Automatic crack detection in built infrastructure using unmanned aerial vehicles," arXiv preprint arXiv:1707.09715, 2017.

[21] J. Casper and R. R. Murphy, "Human-robot interactions during the robot-assisted urban search and rescue response at the world trade center," IEEE Transactions on Systems, Man, and Cybernetics, Part B (Cybernetics), vol. 33, no. 3, pp. 367-385, 2003.

[22] N. Simaan, R. Taylor, and P. Flint, "A dexterous system for laryngeal surgery," in Robotics and Automation, 2004. Proceedings. ICRA'04. 2004 IEEE International Conference on, vol. 1, pp. 351-357, IEEE, 2004.

[23] C. A. Klein, C. Chu-Jenq, and S. Ahmed, "A new formulation of the extended jacobian method and its use in mapping algorithmic singularities for kinematically redundant manipulators," IEEE Transactions on Robotics and Automation, vol. 11, no. 1, pp. 50-55, 1995.

[24] P.-H. Chang, K. C. Park, and S. Lee, "An extension to operational space for kinematically redundant manipulators: kinematics and dynamics," IEEE Transactions on Robotics and Automation, vol. 16, no. 5, pp. 592-596, 2000.

[25] B. Bayle, J.-Y. Fourquet, and M. Renaud, "Manipulability analysis for mobile manipulators," in Robotics and Automation, 2001. Proceedings 2001 ICRA. IEEE International Conference on, vol. 2, pp. 1251-1256, IEEE, 2001.

[26] A. Melingui, J. J.-B. M. Ahanda, O. Lakhal, J. B. Mbede, and R. Merzouki, "Adaptive algorithms for performance improvement of a class of continuum manipulators," IEEE Transactions on Systems, Man, and Cybernetics: Systems, 2017.

[27] O. Lakhal, A. Melingui, and R. Merzouki, "Hybrid approach for modeling and solving of kinematics of a compact bionic handling assistant manipulator," IEEE/ASME Transactions on Mechatronics, vol. 21, no. 3, pp. 1326-1335, 2016.

[28] A. Melingui, O. Lakhal, B. Daachi, J. B. Mbede, and R. Merzouki, "Adaptive neural network control of a compact bionic handling arm," IEEE/ASME Transactions on Mechatronics, vol. 20, no. 6, pp. 2862$2875,2015$.

[29] O. Lakhal, A. Melingui, T. Morales, C. Escande, and R. Merzouki, "Forward kinematic of a class of continuum bionic handling arm," Accepted at Robotics and Biomimetics (ROBIO), 2014 IEEE International Conference on, 2014.

[30] W. Khalil and J. Kleinfinger, "A new geometric notation for open and closed-loop robots," in Robotics and Automation. Proceedings. 1986 IEEE International Conference on, vol. 3, pp. 1174-1179, IEEE, 1986.

[31] J. Denhavit, "A kinematic notation for lower-pair mechanisms based on matrices," ASME J. Appl. Mech., 1955.

[32] R. P. Paul, Robot manipulators: mathematics, programming, and control: the computer control of robot manipulators. Richard Paul, 1981.

[33] M. Frank and P. Wolfe, "An algorithm for quadratic programming,", Naval research logistics quarterly, vol. 3, no. 1-2, pp. 95-110, 1956.

[34] M. Karacan and B. Bükcü, "On natural curvatures of bishop frame," Journal of Vectorial Relativity, vol. 5, pp. 34-41, 2010.

[35] A. J. Hanson and H. Ma, "Parallel transport approach to curve framing," vol. 11, pp. 3-7, 1995. 